\title{
SPEAR2 RF OPERATION: A BRIDGE TO SPEAR3*
}

\author{
Sanghyun Park \\ Stanford Linear Accelerator Center, P.O. Box 4349, Stanford, California 94309, USA
}

\begin{abstract}
The present schedule calls for the Spear2 RF system operation until March of 2003, when it will be dismantled to make room for the Spear3. While any new investment to improve the system is discouraged for this limited-time operation, efforts are focused on maintaining the system reliability and accommodating any changes necessitated by new construction in preparation for the Spear3. In this paper we will present our operational experience with the changes incorporated into the system. The Booster RF system improvement to raise the injection energy from 2.3 to $3.0 \mathrm{GeV}$ will be also discussed.
\end{abstract}

\section{INTRODUCTION}

The SPEAR (Stanford Positron Electron Asymmetric Ring) RF system was closely modeled after the PEP-I system for its operation as a light source. With the RF frequency being so close to each other (Spear2 at 358.54 $\mathrm{MHz}$, PEP-I at $352.00 \mathrm{MHz}$ ), the low-level RF (LLRF) systems are identical up to the klystron RF input port. The Spear 2 cavities are re-tuned version of the PEP-I cavities, whose nosecones were reshaped for the right frequency.

The Spear3 is a major upgrade[1] for higher current and much lower emittance, leading to a higher brightness of the photon flux. This goal is achieved through a number of changes in the magnetic lattice as well as the RF system upgrade to higher power klystron, HOM-damped single-cell cavities, and digital control of the LLRF. From the hardware point of view, the Spear3 RF is $1 / 3$ of the PEP-II high-energy ring (HER) RF station at IR8. At HER, $1000 \mathrm{~mA}$ of $9 \mathrm{GeV}$ electron beam is stored. The Spear3 RF HVPS is identical to the PEP-II supplies. Thus the whole system is operated and maintained by the same procedure used at PEP-II. What this means is that the spare can be shared between the two systems, giving benefits to both systems in case of any system failure.

The booster synchrotron RF system will not be changed except that the driving RF frequency of $358.5 \mathrm{MHz}$ is to be modulated by up to $7.0 \mathrm{kHz}$ during the energy ramp so the system stays phase-locked with the Spear3. Otherwise the Spear3 buckets are randomly filled. The injector linac and the thermionic cathode RF gun are to be maintained at the present setting. The $105 \mathrm{MeV}$ linac beam energy will remain the same. At the RF gun, the thermionic cathode continues to be a subject of an active study for better operational stability, and to achieve longer service life.

\section{OPERATIONAL EXPERIENCE}

The Spear2 has two identical and independent RF systems. One is in operation, the other in standby mode. Each consists of one RFHVPS powering one klystron that energizes one 5-cell cavity. The operational parameters such as DC power and RF power have been much lower than the design spec of the high-power subsystems such as PS, klystron, and cavity. This contributed to their long life and better reliability by virtue of lower stress.

During the FY99 user run, one RF system experienced difficulties in turning it on for high-power operation. First it would not process up to the normal 1.6 MV gap voltage. Then the system had frequent trips on excessive reflected power off the cavity, accompanied by a loud arcing noise. The other system was put into service for the rest of the user run. Along the way the working PS failed. The other PS was turned on to power the active klystron temporarily until the user run was over in summer.[2]

When the failed cavity was opened up it was discovered that a vacuum valve nylon washer made its way to the cavity bottom, outgassing and turning itself to carbon during the RF processing. The subsequent arcing was from a displaced Metex ${ }^{\circledR}$ seal at the window bottom airside, in the waveguide-to-coax transition. Both cavities were thoroughly cleaned and nitrided. The movable tuner assemblies were mechanically reinforced to improve the stability. Graphite dusts on the tuner slugs were cleaned and the tuner shafts were polished not to scrape off the sliding graphite fingers, which block cavity RF power from going into the tuner bellow.

The waveguides are maintained at a positive pressure by dry air to keep the moisture out of the RF power and to let the system trip in case any opening develops and the RF power leaks out along with the air. Without the benefit of a circulator, the klystron and the cavity are directly coupled. This sometimes leads to beam instability. If the differential pressure is adjusted to a certain set point by a feedback control, the RF phase delay can be controlled (by changing the waveguide cross section) to the extent that the coupling and beam instability are minimized. This scheme was successfully implemented to both systems.

*Work supported by USDoE contract DE-AC03-76SF00515

Presented at the IEEE Particle Accelerator Conference (PAC 2001), 6/18/2001- 6/22/2001, Chicago, IL 
There have been numerous attempts to improve on the beam lifetime by changing the filling pattern. The best pattern often brought in beam instability caused by HOM. Some modes were visible at the waveguide directional coupler and there was no damper. In the summer of 2000 , the mitre bend nearest to the cavity was modified to filter out and dissipated the HOM by ferrite tiles soldered on a thick copper plate, which is water-cooled. With the HOM damper installed, a remarkable increase in beam lifetime was achieved without causing any beam instability. At the active system the nearest bend is an H-mitre, whereas the other system has an E-mitre. HFSS simulation was used to design both types of damper. Network analyzer tests did confirm the simulation results. [3]

\section{SPEAR3 RF SYSTEM}

When the Spear3 upgrade project was being proposed, a number of different cavity types were studied for its possible adaptation to the Spear3 RF system to sustain the stored beam of $500 \mathrm{~mA}$ at $3.0 \mathrm{GeV}$, with a provision of going higher in the beam energy to $3.3 \mathrm{GeV}$. With a set of constraints given in terms of the circumference and the short section lengths, the decision was to follow the PEPII RF system for the reasons described below.

Recycling of the Spear2 5-cell cavities was considered and rejected for they have lower power rating $(\sim 250 \mathrm{~kW}$ each) and stronger HOM intensities at high current stored. Two of those cavities would have sustained only up to 200 $\mathrm{mA}$ at $3 \mathrm{GeV}$, and even lower current at higher energies. Then three types of single-cell cavities were studied. They are cavities at Photon Factory, APS, and PEP-II at SLAC B-factory. Initially all these were to be scaled to Spear2 frequency at $358.54 \mathrm{MHz}$ since the Spear3 upgrade leaves the circumference essentially unchanged at 234.12 meters.

The APS and PF cavities have simple structure, making it easy to scale up (from 352 APS) or scale down (from $500 \mathrm{PF}$ ) to $358 \mathrm{MHz}$ Spear frequency. The APS cavity is to take up considerably smaller space. The concern was that HOM damping was not sufficient for Spear3 $500 \mathrm{~mA}$ operation. The PEP-II cavity, on the other hand, has very good HOM damping while it has complicated structure. It takes considerable engineering efforts to scale down to the present Spear frequency. [5,6]

In order to satisfy the constraints of Spear and Booster circumferences, and to fit into the tight schedule, PEP-II cavity operating at slightly higher frequency $(476.3 \mathrm{MHz})$ was selected. The cavities are currently being fabricated at the vendor facility. Each cavity assembly consists of one bare cavity, ceramic aperture window, matching network, waveguide HOM load, a vacuum pump, and three HOM loads in folded waveguide attached to the cavity. Spear3 employs four such assemblies in a straight section of the ring previously occupied by high-energy physics detector.
One important consideration in selecting PEP-II cavity is interchangeability of most of the subsystems between the two systems. For example, one klystron purchased for Spear3 is already in service at the PEP-II HER RF station. In return, Spear3 will use a higher-power rated klystron being built onsite. Klystron, RFHVPS, circulator, and water-loads are fully compatible. Even the RF cavity is interchangeable with movable tuner position adjusted: the Spear3 cavity can be used when the tuner is pulled out, and vice versa.

The Spear3 beam and RF parameters are summarized in Table 1, in comparison with Spear2 [4].

Table 1. Spear2 and Spear3 beam and RF parameters.

\begin{tabular}{|l|c|c|c|}
\hline Parameter & unit & Spear2 & Spear3 \\
\hline \hline Beam Energy & $\mathrm{GeV}$ & 3.0 & 3.0 \\
Beam Current & $\mathrm{mA}$ & 100 & 500 \\
Emittance & $\mathrm{nm}-\mathrm{rad}$ & 160 & 18 \\
Lattice type & & FODO & DBA \\
Bend radius & $\mathrm{m}$ & 12.47 & 7.858 \\
RF frequency & $\mathrm{MHz}$ & 358.5 & 476.3 \\
Gap voltage & $\mathrm{MV}$ & 1.6 & 3.2 \\
RF power & $\mathrm{kW}$ & 200 & 1,200 \\
Cavity type & & $5-$ cell & single-cell \\
No. cavity & & 1 & 4 \\
Inject. energy & $\mathrm{GeV}$ & 2.3 & 3.0 \\
\hline
\end{tabular}

The reduction of beam emittance by nearly two orders of magnitude is due to the change in magnetic lattice from FODO to double bend achromat (DBA). The RF power is increased by a factor of 6 , to store higher current in tighter bend. If insertion devices are added on as planned, the RF power will be saturated at $1.20 \mathrm{MW}$ by 2008 . Then either the klystron is upgraded, or the beam current is reduced accordingly. By 2022, all the available straight sections will be occupied by ID's and 1.29 MW of RF power will be needed to sustain $500 \mathrm{~mA}$. Then as a $10 \%$ operational margin is added, a total of $1.42 \mathrm{MW}$ klystron output is needed. The original PEP-II klystron (there is one unit in operation and another in the making) built by SLAC is capable of generating that much power.

Acquisition process is presently underway for all other high-power subsystems such as power supply, circulator, magic-T, and water-loads. The new RF station is to be located near the West-pit where four single-cell cavities will be installed. The new power supply of $90 \mathrm{kV}$ at $27 \mathrm{~A}$ is to be housed in the present PS cage, where two Spear2 power supplies $(66 \mathrm{kV}, 13 \mathrm{~A}$ each) are located. One spare PS is to be replaced by a new one in the summer of 2002 to save time for installation of the new systems in 2003. The HCW (high conductivity water) station is also being worked on for the 4 high-power RF water loads, one at the circulator and one each at the 3 units of magic-T. 
The Spear3 will use essentially the same LLRF that is in operation at PEP-II. Since PEP-II already experiences difficulties in purchasing spare components that became obsolete, the PEP-II efforts to improve LLRF in terms of parts availability and serviceability will benefit Spear3. Any improvement in the system performance at PEP-II is to be directly applied to the Spear3 version. [7]

\section{BOOSTER FOR SPEAR3}

As explained earlier, the Spear3 RF system is new in every respect. The Booster RF hardware, however, is to remain the same except that its operation will be changed: higher gap voltage for $3.0 \mathrm{GeV}$ injection energy and slight change in frequency (with up to $7 \mathrm{kHz}$ modulation) to stay in phase with the Spear3 RF. The Booster RF parameters are summarized in the table below.

Table 2. Booster RF parameters at Spear2 and Spear3.

\begin{tabular}{|l|c|c|c|}
\hline Parameters & Unit & @ Spear2 & @ Spear3 \\
\hline \hline Booster frequency $\left(\mathrm{f}_{\mathrm{B}}\right)$ & $\mathrm{MHz}$ & 358.540 & 358.505 \\
Spear frequency $\left(\mathrm{f}_{\mathrm{s}}\right)$ & $\mathrm{MHz}$ & 358.540 & 476.300 \\
Frequency ratio $\left(\mathrm{f}_{\mathrm{S}}: \mathrm{f}_{\mathrm{B}}\right)$ & & $1: 1$ & $97: 70$ \\
Harmonic no. ratio $(\mathrm{h}: \mathrm{h})$ & & $280: 160$ & $372: 160$ \\
Booster freq. modulation & $\mathrm{kHz}$ & 0 & $\leq 7.0$ \\
Booster peak gap voltage & $\mathrm{MV}$ & 0.6 & 1.2 \\
\hline
\end{tabular}

The ratio between the harmonic numbers 280 (Spear2) and 160 (Booster) is 7 to 4 . In every $3.12 \mu \mathrm{s}$, the target Spear bucket to be filled comes in phase with the Booster bunch. Booster is phase-locked to Spear2 RF frequency. If the Booster bunch fails to reach the designated Spear RF bucket for whatever reasons, it has another chance $3.12 \mu \mathrm{s}$ later. The bunch energy doesn't change very much over this short period of time since the bending magnet current is near its maximum in the sinusoidal waveform.

With the Spear3, however, the ratio is 97/70. Booster's bunch is phased with a given Spear bucket every $54.6 \mu \mathrm{s}$, which is too long for the bunch energy to stay about the same. To improve on the matching, suppose each Booster $\mathrm{RF}$ bucket is delayed in phase. During one revolution at the Spear3, Booster RF would have

$$
\Delta \phi=360^{\circ} \times(358.505 \mathrm{MHz}) \times(0.781020 \mu \mathrm{s})=100,800^{\circ}
$$

This corresponds to the frequency shift, $\Delta \mathrm{f}$, of

$$
\Delta \mathrm{f}=\Delta \phi / \Delta \mathrm{t}=7.0 \mathrm{kHz}
$$

where $\Delta \phi=100,800^{\circ}=280$ periods, $\Delta \mathrm{t}=40 \mathrm{~ms}$ in Booster is how long the bunch is accelerated. Now that this is the maximum phase shift needed to align the Booster bunch to a designated Spear3 RF bucket, Booster RF frequency of $358.505 \mathrm{MHz}$ must be modulated by not more than 7.0 $\mathrm{kHz}$ over the $40 \mathrm{~ms}$ of the Booster bunch acceleration. Details of Spear2-Booster-Linac timing system have been described elsewhere[8] in terms of fiducial timing pulse, modulator switching, kicker triggers, etc.

The master oscillator generates the Spear3 frequency at 476.300 MHz. The frequency source has been installed, set at the Spear2 frequency to observe its stability. On a digital frequency meter, the reading is stable within $1 \mathrm{~Hz}$ over a week of operation. This frequency is divided down by 93 , and multiplied up by 70 to produce the Booster reference frequency at $358.505 \mathrm{MHz}$. Various frequencies are derived from the master oscillator to produce system clock, BPM LO signal, BPM ADC sampling, and so forth.

The Booster has been injecting $2.3 \mathrm{GeV}$ beam to Spear for the last 10 years. For a $3.0 \mathrm{GeV}$ injection, the cavity gap voltage must be raised accordingly. One estimate puts the needed peak gap voltage at $0.8 \mathrm{MV}$, which is routinely set during the RF processing following annual shutdowns. A test is scheduled at the end of user run to verify the gap voltage requirement.

\section{INJECTOR LINAC}

The injector linac and the thermionic cathode RF gun configuration will not be changed. The S-band klystron, modulator, drive amplifier, and the linac master oscillator will stay the same. There are adequate level of spares and spare parts readily available in case of any emergencies. Some efforts were made to streamline the inventories for GTF and injector linac systems so that the former uses the subsystems that are the spare for the latter[9].

The thermionic cathode, however, demands continued attention. During the last two weeks of January 2001, the RF gun cathode heater resistance decreased continuously. Its electrical characteristics are closely monitored. Those seem to be flattened out for the last several months. During the 2001 summer shutdown, the heater AC power supply will be worked on to improve its modularity for a quick repair in case of failure, and for better diagnostics.

\section{REFERENCES}

[1] J. Corbett, et al., These Proceedings R. Hettel, et al., EPAC2000 Proc

[2] S. Park, CWRF2000 (ANL/APS) Proc., unpublished

[3] J. Sebek, et al., These Proceedings

[4] R. Hettel, et al., PAC91 Proc

[5] S. Park, PAC99 Proc.

[6] R. Rimmer, et al., PAC95 Proc.

[7] P. Corredoura, et al., PAC95 Proc.

[8] R. Hettel, et al., PAC91 Proc.

[9] J. Weaver, et al., PAC91 Proc.; S. Park, Linac98 and Linac2000 Proc. 